\title{
COVID-19: Of Schrödinger, cats and masks
}

\author{
George Thomson ${ }^{1,2}$ \\ ${ }^{1}$ International Journal of Clinical Practice Open Research \\ ${ }^{2}$ Royal Cornwall Hospital
}

April 28, 2020

\begin{abstract}
COVID-19 is a disease which is sweeping the globe, often with devastating consequences. The more we understand, the more it appears that infection has a very wide clinical spectrum from totally asymptomatic to life threatening. Without widespread community testing it is impossible to ascertain true infection rates and develop strategies which reduce or prevent transmission without the need for on-going draconian measures such as complete national lockdown. These measures are mandatory at the time of writing, however widespread adoption of face masks has been shown to help prevent transmission of other respiratory pathogens, and also infections acquired by healthcare staff. Combining mass testing with a combination of social distancing and face mask use might offer a way forward until a mass COVID-19 vaccination programme can be established.
\end{abstract}

The COVID-19 pandemic progresses across the globe at pace, bringing devastating consequences and lockdown of countries and continents. Looking for some brief distraction from the daily news reports, I was drawn to recent apparently random observations that SARS-COV-2 can infect other animal species, especially cats. On 6 April 2020 it was reported that a number of big cats at the Bronx zoo had developed respiratory symptoms, and that a tiger named Nadia had been tested positive for a COVID-19 $(1,2)$. It is assumed that the infection was transmitted by a zookeeper who had fallen ill with COVID-19 and been in contact with the tiger (2). Who was brave enough to take a throat swab from a tiger was not revealed.

Recent evidence suggests that cats (but much less so dogs) can be susceptible to infection with COVID-19, and that the SARS-Cov-2 virus replicates efficiently in cats and ferrets (3). Unlike the big cats at Bronx zoo the initial infection was artificially induced by large doses of SARS-Cov-2, and did not result from exposure to a single infected human. This study demonstrated that the infected cats were asymptomatic but were able to infect other nearby cats through droplets spread thus maintaining the infection chain. The observation has led the Center for diseases control (CDC) to advise people with symptoms of COVID-19 to minimise contact with their pets, including petting, snuggling, and being licked (2). It should be noted that as yet there is no evidence of pet to human transmission, nor whether cats go on to develop immunity, but it is hard to ignore the possibility that our pets might act as reservoirs for SARS-Cov-2. Notwithstanding the possibility that in the months to come there may be herds of cats immune to COVID-19, it is hard to escape the likelihood that until either human herd immunity or mass vaccination against COVID-19 becomes a reality, the SARS-Cov-2 virus is likely to live on in our communities for some time to come. This is likely to have implications with respect to the need for some form of ongoing non-pharmaceutical interventions for the foreseeable future.

The recent observations of COVID-19 infection in cats brought to mind recent social media posts remembering Erwin Schrodinger's "cat paradox paper" (4) - colloquially referred to as Schrodinger's cat. Given the lack of mass community testing for SARS-Cov-2 in most countries to date, the posts suggested that COVID-19 could be considered to be the 'Schrodinger's virus'. Unfortunately, it is not possible to cite an original author as the nature of social media posts usually obscure the origin, but whoever came up with the concept in to be commended, and if they can be identified should take significant credit. 
In 1935, Schrodinger attempted to describe a paradox in quantum mechanics which suggests that physical objects can simultaneously exist in any possible state at any given point in time. The paradox was postulated by Einstein, and Schrodinger sought to explain the concept to those with the more average mind. Briefly, he described a cat placed in a sealed box in which the observer could not see the cat. In the box was a vial of deadly poison gas, which would be released upon the random degeneration of a radioactive substance. He explained that at any given time, the cat will either be alive or dead, but the observer would not know which state existed for the cat. Therefore, the cat must be assumed to be both alive and dead at the same time. Whilst Schrodinger's concept may seem macabre, it has great relevance to COVID-19 in a situation where scientists remain in the dark as to the actual extent of community wide infection rates.

Likening Schrödinger's cat to 'Schrodinger's virus' is therefore highly relevant. There is good evidence that people infected with COVID-19, who by definition must be considered to be infectious, are often asymptomatic (5) or pre-symptomatic (6). Without regular mass testing of whole populations it is thus impossible to know in what state (previously infected and thus probably immune, currently infected, or not infected) anyone we meet exists. This applies to both healthcare setting interactions and also any person-to-person interaction in the community - hence 'Schrodinger's virus'. Therefore, in light of a lack of widespread testing, every human to human interaction has to be assumed to be both an opportunity to infect the other person or be infected by them.

This paradox emphasises the need for global leaders of health, national and international policy, to urgently address what measures are necessary to prevent the further spread of COVID-19. National and international lockdown policies cannot stay in place forever, and many are now discussing exit strategies. A recent paper, yet to be peer reviewed, but already shared via the SSRN community (7), explores the impact of nationwide policies for wearing a face mask in public. The authors point out that the data on COVID-19 to date show that in countries (all SE Asian) where the universal wearing of facemasks is the norm, there is a significant reduction in average daily growth rates of confirmed COVID-19 infection (10\% versus $18 \%$ ). They also model substantial societal and financial benefits through reduced COVID-19 transmission, healthcare burden, suffering and deaths. It is important to note that their modelling is based on the use of home-made cloth face masks, preserving medical masks for frontline health and social care staff. The CDC in the United States have also released a recommendation on the use of cloth face masks in public settings (6).

There has been debate for years on the effectiveness of facemasks to prevent viral transmission in both community and healthcare settings (8). However, notwithstanding the data on COVID-19 infection rates highlighted recently $(6,7)$ there are now fairly compelling data to support widespread use of facemasks. If used consistently, as is likely at a time of pandemic, masks have been shown to significantly reduce transmission from people infected with respiratory pathogens to others (9) with a reduction of 3.4 fold in viral aerosol shedding of influenza virus demonstrated through the use of facemasks in another study (10). For healthcare workers much emphasis has been placed on the difference between aerosol and droplet spread and the lack of evidence for face masks with the latter. However, investigators studying the potential benefit of N95 masks versus conventional medical masks concluded that this distinction between aerosol and droplets was an oversimplification (11). They showed that N95 masks, which are masks that are tight fitting and filtered, provided significantly greater protection than standard medical masks against contracting respiratory infection in healthcare workers. Importantly, the relative risk of laboratory confirmed viral infections overall was reduced to just 0.46 (95\% CI 0.23-0.91) when compared with medical masks, and droplet transmission had an even greater reduction in relative risk to just 0.26 (95\% CI 0.16-0.42) using N95 masks.

The world will inevitably move towards some form of relaxation of lockdown in the not too distant future, and this is important for societal, mental health, and economic reasons. However, it is important that COVID19 does not re-emerge in epidemic proportions again as a result, with similar devastating consequences to those that we are seeing at present. There appears to be a strong body of evidence, and also guidance from respected international authorities to support the use of simple, society wide home-made face masks, and the use of better protection for healthcare workers exposed to the risk of COVID-19. Hopefully, combined with ongoing social distancing measures, and availability of widespread testing facilities to support isolated 
quarantine, such an approach might mitigate against the resurgence of COVID-19 as lockdown measures are relaxed.

There is also a strong case for a thorough evidence based global review of which masks should be used when, and in what settings. Perhaps, were Schrödinger's cat alive, and obviously dead at the same time, in the age of COVID-19 it would have chosen to wear a mask and also preserve a typically indifferent feline distance from strangers.

\section{References}

1. BBC News. Coronavirus: Tiger at Bronx Zoo tests positive for Covid-19. 2020, https://www.bbc.co.uk/news/worldus-canada-52177586

2. Centers for disease control and prevention. Coronavirus Disease 2019 (COVID-19). 2020, https://www.cdc.gov/coronavir ncov/daily-life-coping/animals.html

3. Shi J, Wen Z, Zhong G, et. al. Susceptibility of ferrets, cats, dogs, and different domestic animals to SARS-coronavirus-2. 2020. doi: https://doi.org/10.1101/2020.03.30.015347

4. Schrödinger E. The present situation in quantum mechanics: a translation of Schrodingers "cat paradox paper". Proc. Am. Phil. Soc., 124, 323-38.

5. Mizumoto K, Kagaya K, Zarebski A, Chowell G. Estimating the asymptomatic proportion of coronavirus disease 2019 (COVID-19) cases on board the Diamond Princess cruise ship, Yokohama, Japan, 2020. 2020, Euro Surveill. 25(10), 2-7. doi: 10.2807/1560-7917.ES.2020.25.10.2000180.

6. Centers for disease control. Recommendation Regarding the Use of Cloth Face Coverings, Especially in Areas of Significant Community-Based Transmission. 2020, https://www.cdc.gov/coronavirus/2019ncov/prevent-getting-sick/cloth-face-cover.html\#studies

7. Christakis NA, Abaluck J, Chevalier J, et al. The Case for Universal Cloth Mask Adoption \& Policies to Increase the Supply of Medical Masks for Health Workers April 12020 https://papers.ssrn.com/sol3/papers.cfm?abstrac $\mathrm{id}=3567438$ [Epub ahead of print]

8. Bin-Reza F, Lopez Chavarrias V, Nicoll A, Chamberland ME. The use of masks and respirators to prevent transmission of influenza: a systematic review of the scientific evidence. 2012. Influenza Other Respir Viruses. 6(4):257-67. doi: 10.1111/j.1750-2659.2011.00307.x. Epub 2011 Dec 21.

9. MacIntyre CR, Cauchemez S, Dwyer DE, et. al. Face mask use and control of respiratory virus transmission in households. 2009. Emerg Infect Dis. 15(2):233-41.

10. Milton DK ${ }^{1}$, Fabian MP, Cowling BJ, Grantham ML, McDevitt JJ. Influenza virus aerosols in human exhaled breath: particle size, culturability, and effect of surgical masks. 2013 PLoS Pathog. 9(3):e1003205. doi: 10.1371/journal.ppat.1003205.

11. MacIntyre CR, Chughtai AA, Rahman B, Peng Y, Zhang Y, Seale H, Wang X, Wang Q. The efficacy of medical masks and respirators against respiratory infection in healthcare workers. 2017. Influenza Other Respir Viruses. 11(6):511-517. doi: 10.1111/irv.12474. 